\title{
Deriving high-resolution velocity maps from low-resolution Fourier velocity encoded MRI data
}

Vinicius de Carvalho Rispoli (vrispoli@pgea.unb.br) Joao Luiz Azevedo de Carvalho (joaoluiz@pgea.unb.br)

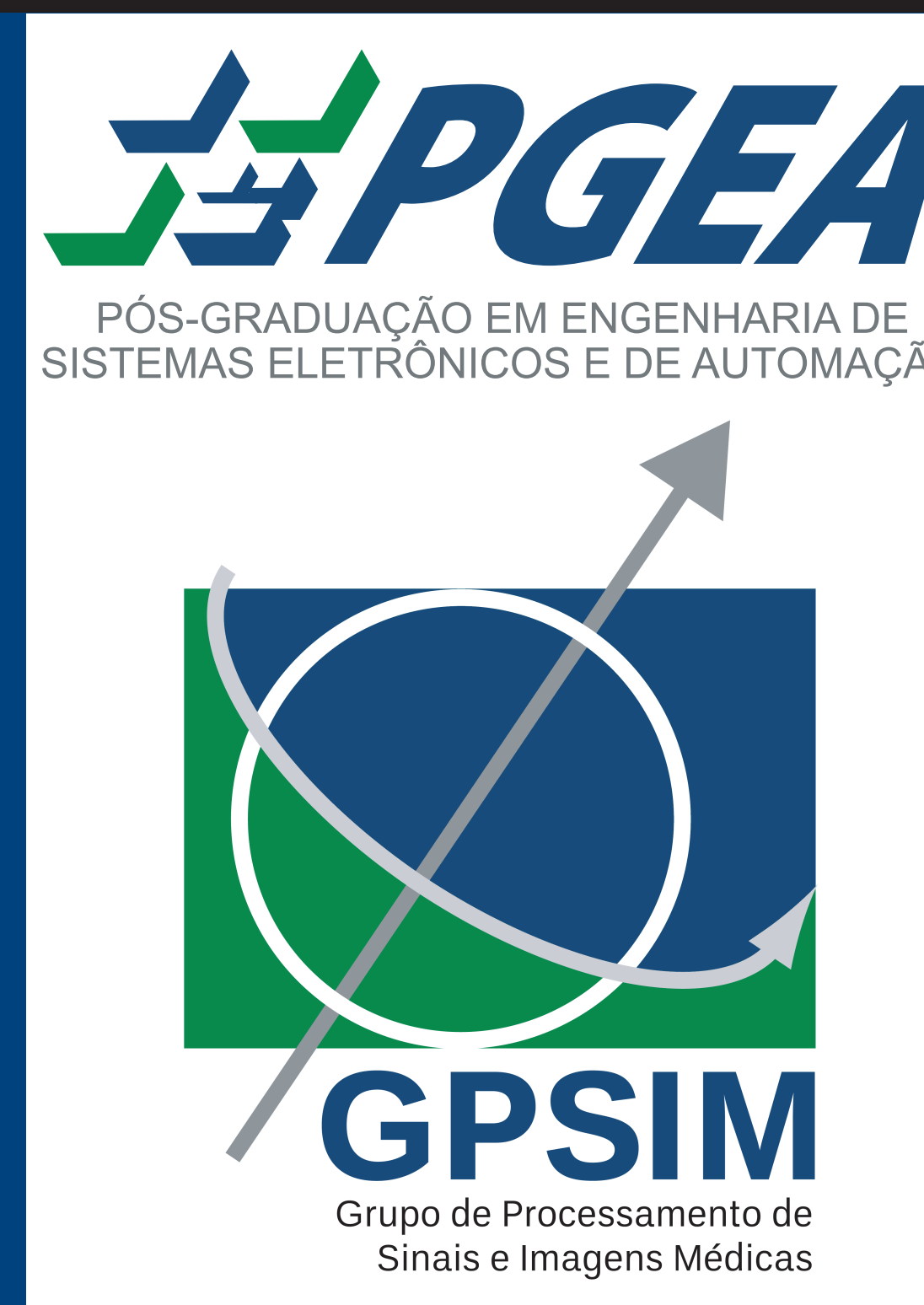

(b)

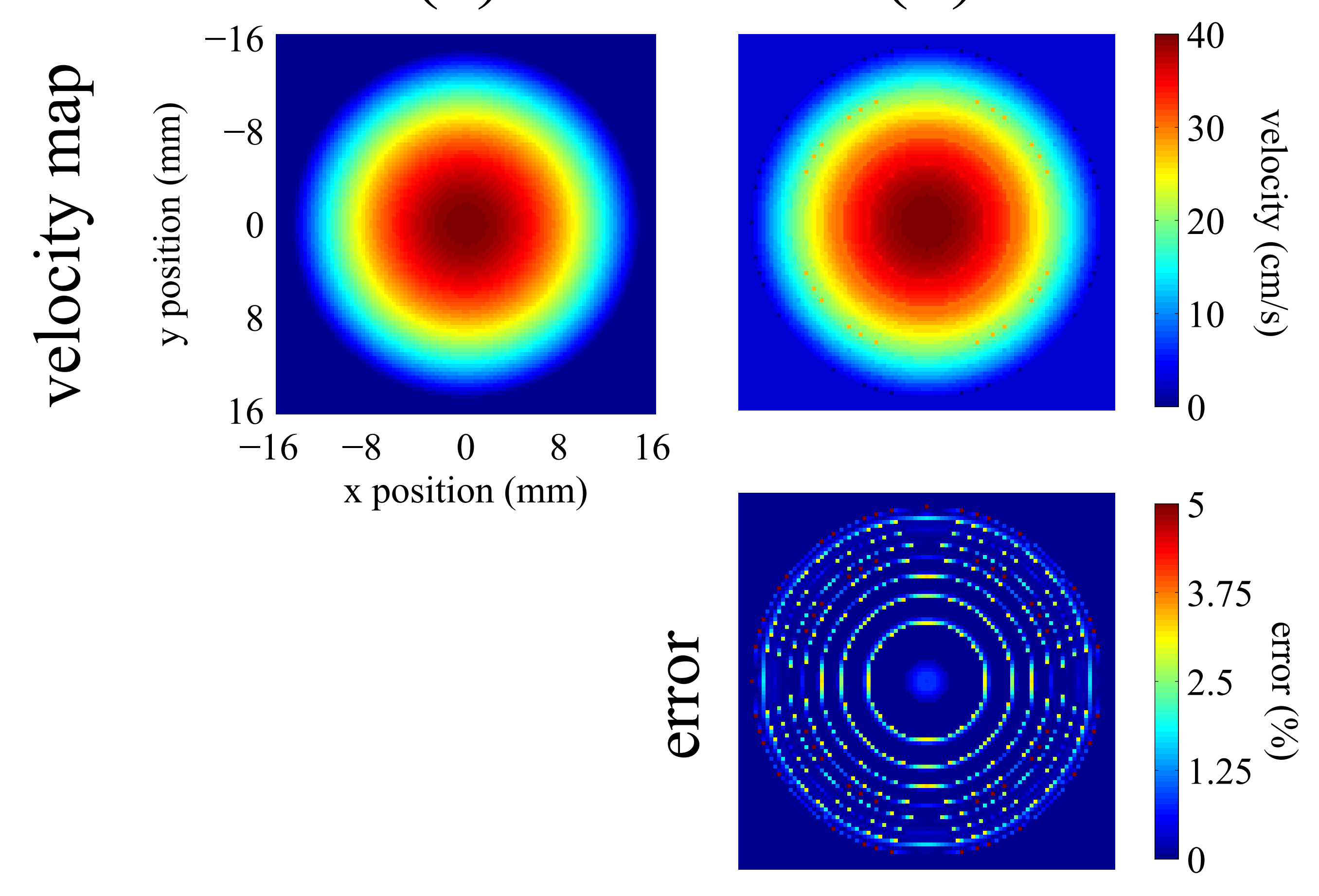

Figure 3: Experiment using a numerical phantom of parabolic flow: (a) reference velocity map; (b) velocity map estimated from the simulated low-resolution FVE data, and associated

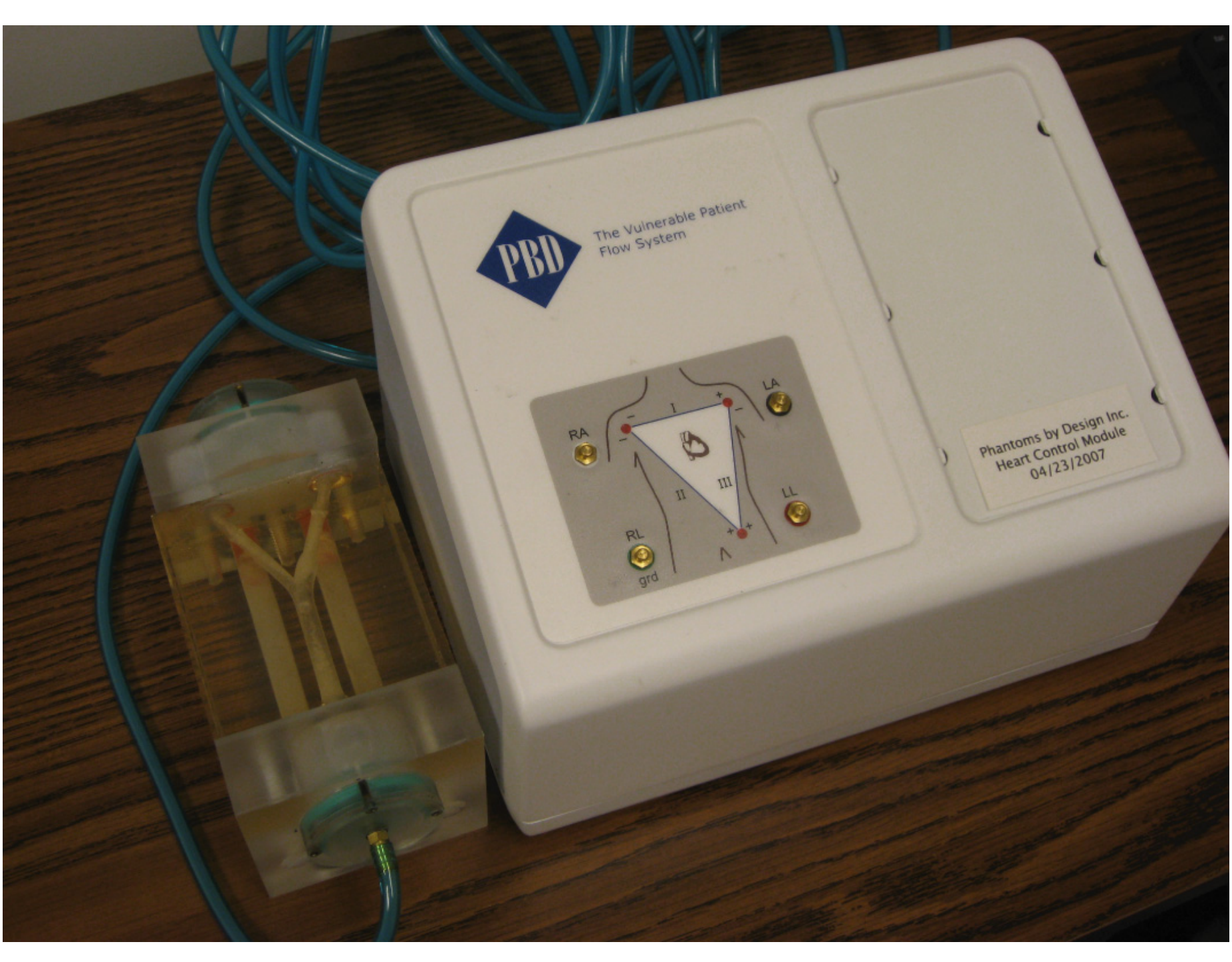

posed in ref. [4]. Hence, we obtain:

$$
\tilde{s}(x, y, v) \approx m(x, y) \cdot \operatorname{sinc}\left(\frac{v-v_{o}(x, y)}{\Delta v}\right)
$$

- If a high-resolution spin-density map, $\tilde{m}(x, y)$, is available, the velocity $v_{o}$ associated with a given pixel $\left(x_{0}, y_{0}\right)$ may be estimated from $\tilde{s}(x, y, v)$ as:

$$
\tilde{v}_{o}\left(x_{o}, y_{o}\right)=\arg \min _{\nu}\left\|\frac{\tilde{s}\left(x_{o}, y_{o}, v\right)}{\tilde{m}\left(x_{o}, y_{o}\right)}-\operatorname{sinc}\left(\frac{v-\nu}{\Delta v}\right)\right\|_{2}
$$

Figure 2: Pulsatile carotid flow phantom (Phantoms by Design, Inc., Bothell, WA).

Results and discussion

- Experiment 1: The velocity map estimated from the simulated low-resolution FVE data was accurate within $3 \%$ for the vast majority of the pixels (Fig. 3). This is a very important result, as carotid flow distant to the bifurcation is typically approximately parabolic.

- Experiment 2: The maps estimated from the simulated low-resolution FVE data are very similar (qualitatively) to the reference map (Fig. 4). The error images show that the map obtained using spatial deconvolution (Fig. 4c) was more accurate than the one obtained without spatial deconvolution (Fig. 4b).

\section{Conclusion} error percentages. (a)

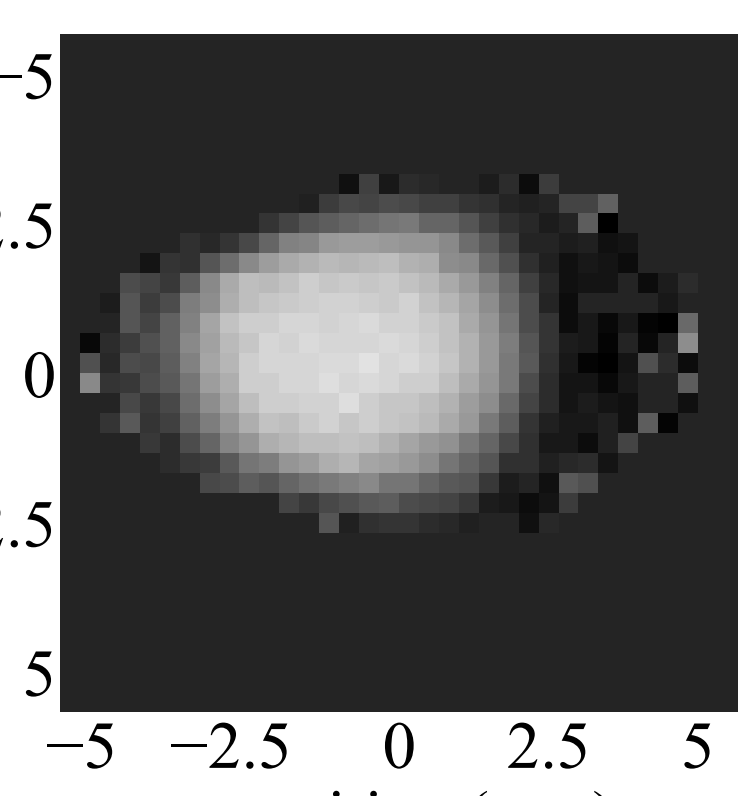

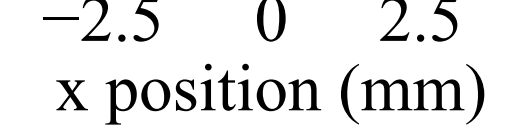

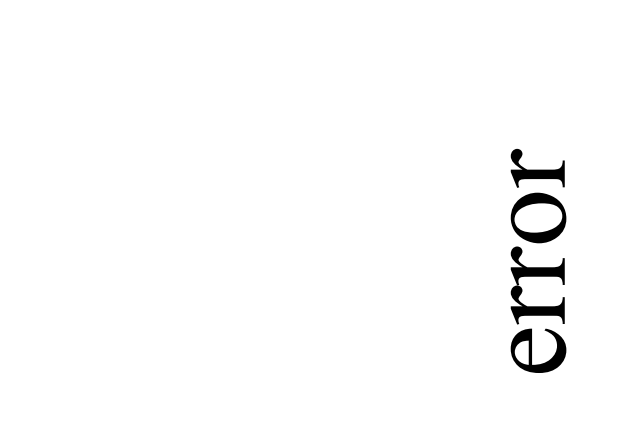

(b)
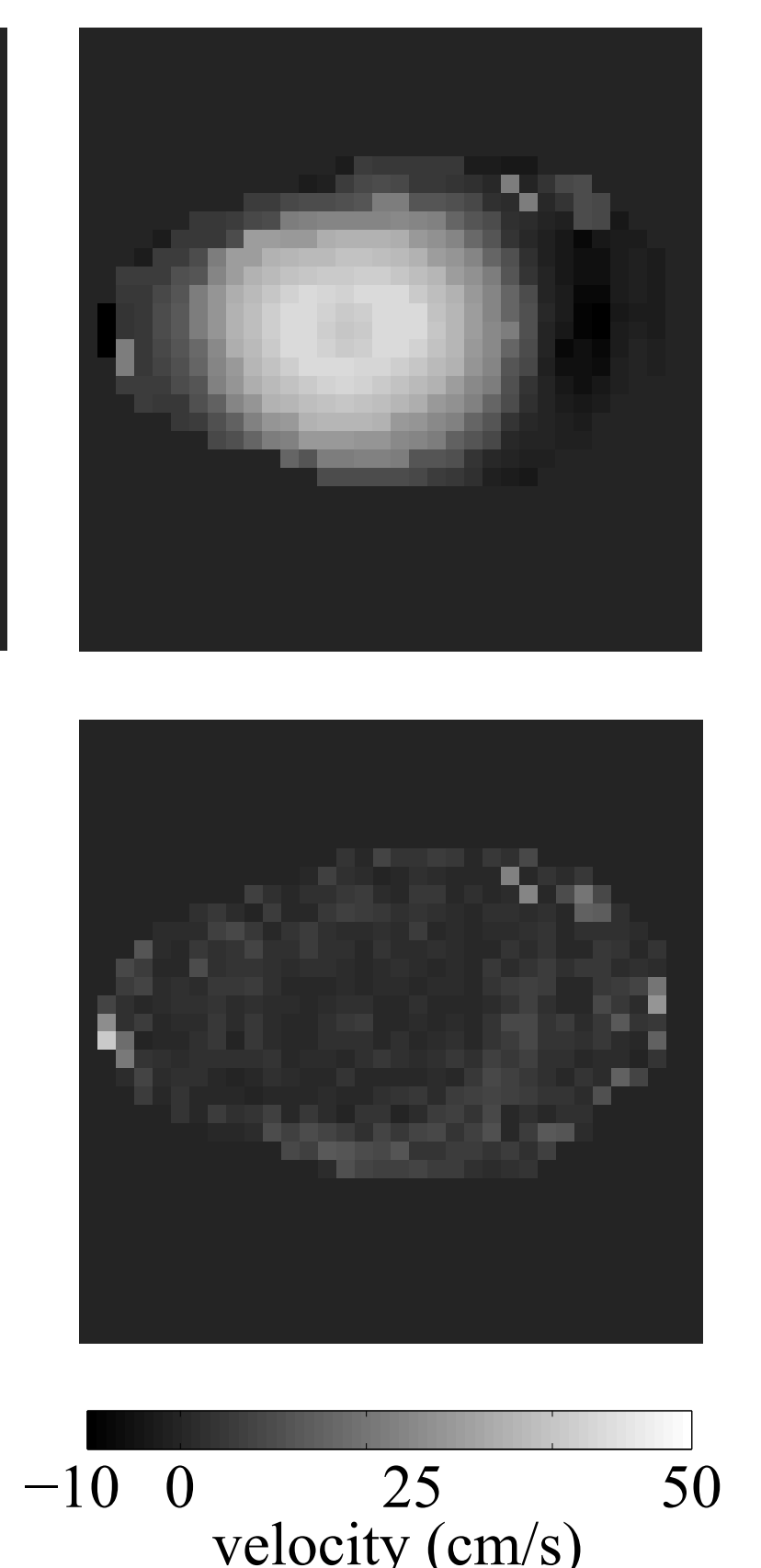

(c)
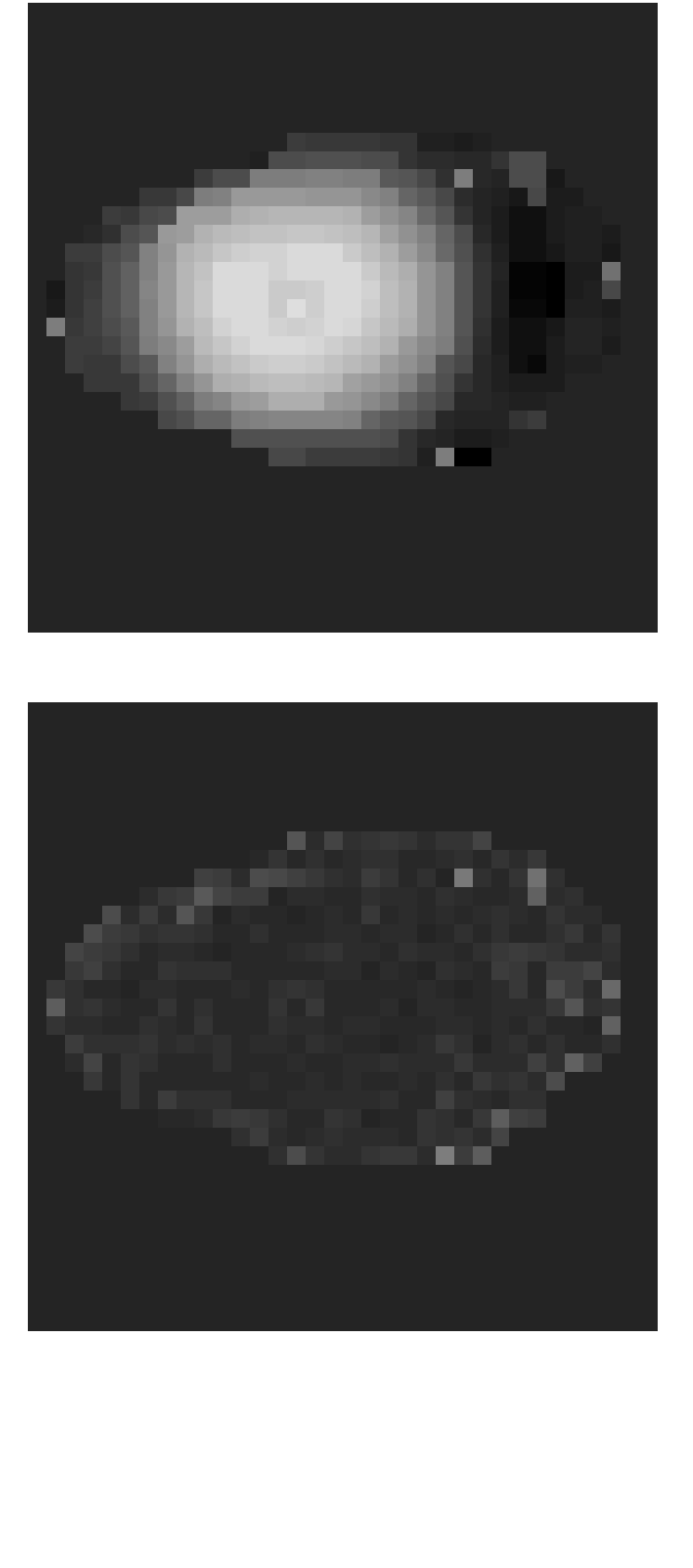

Figure 4: Experiment using the pulsatile carotid flow phantom: (a) reference $P C$ velocity map, measured at the phantom's bifurcation; (b) velocity map estimated from the simulated low-resolution FVE data, without spatial deconvolution (and associated error percentages); and (c) velocity map estimated from the simulated low-resolution FVE data, with spatial deconvolution (and associated error percentages).

\section{References}

[1] Moran PR. MRI 1:197, 1982.

[2] Carvalho JLA and Nayak KS. MRM 57:639, 2007.

[3] Carvalho JLA, et al. MRM 63:1537, 2010.

[4] Krishnan D and Fergus R. Proc 24th NIPS, 2009.

[5] Nielsen J-F and Nayak KS. Proc ISMRM 17: 3858, 2009 .

- It is possible to obtain reasonably accurate velocities maps from low-resolution FVE distributions. - FVE may potentially be used for driving CFD simulations of carotid flow [5], with considerably higher SNR and robustness to partial voluming than PC MRI.
Financial support

- Edital DPP/UnB 10/2011 